\title{
A HARDY-TYPE INEQUALITY WITH AHARONOV-BOHM MAGNETIC FIELD ON THE POINCARÉ DISK
}

\begin{abstract}
Li ZHU
Abstract. A version of Aharonov-Bohm magnetic field on the Poincaré disk is introduced, then a Hardy-type inequality with Aharonov-Bohm magnetic field is proved.

Mathematics subject classification (2010): 26D10, 35H20.

Keywords and phrases: Hardy inequality, Aharonov-Bohm magnetic field, Poincaré disk.
\end{abstract}

\section{REFERENCES}

[1] L. AERMARK AND A. LAPTEV, Hardy's inequality for the Grushin operator with a magnetic field of Aharanov-Bohm type, Algebra i Analiz 23, 2 (2011), 1-8. English transl.: St. Petersburg Math. J. 23, 2 (2012), 203-208.

[2] E. Berchio, D. Ganguly, G. Grillo, Sharp Poincaré-Hardy and Poincaré-Rellich inequalities on the hyperbolic space, J. Funct. Anal. 272, 4 (2017), 1661-1703.

[3] E. Berchio, L. D'Ambrosio, D. Ganguly, G. Grillo, Improved L $L^{p}$-Poincaré inequalities on the hyperbolic space, Nonlinear Anal. 157, (2017), 146-166.

[4] G. CARRON, Inegalites de Hardy sur les varietes Riemanniennes non-compactes, J. Math. Pures Appl. 76, 10 (1997), 883-891.

[5] L. D'Ambrosio, Some Hardy inequalities on the Heisenberg group, Differ. Uravn. 40, 4 (2004), 509-521. English transl.: Differ. Equ. 40, 4 (2004), 552-564.

[6] L. D’Ambrosio, Hardy inequalities related to Grushin type operators, Proc. Amer. Math. Soc. 132, 3 (2004), 725-734.

[7] L. D'Ambrosio, S. Dipierro, Hardy inequalities on Riemannian manifolds and applications, Ann. Ins. H. Poinc. Anal. Non Lin. 31, 3 (2014), 449-475.

[8] J. Dou, Q. GuO, AND P. NiU, Hardy inequalities with remainder terms for the generalized BaouendiGrushin vector fields, Math. Inequal. Appl. 13, 3 (2010), 555-570.

[9] N. GAROFALO, Unique continuation for a class of elliptic operators which degenerate on a manifold of arbitrary codimension, J. Differential Equations 104, 1 (1993), 117-146.

[10] N. Garofallo AND E. LanCONELli, Frequency functions on Heisenberg group, the uncertainty principle and unique continuation, Ann. Inst. Fourier (Grenoble) 40, 2 (1990), 313-356.

[11] I. Kombe, M. OZAYDin, Improved Hardy and Rellich inequalities on Riemannian manifolds, Trans. Amer. Math. Soc. 361, 12 (2009), 6193-6203.

[12] I. Kombe, M. OzAYDin, Hardy-Poincaré, Rellich and uncertainty principle inequalities on Riemannian manifolds, Trans. Amer. Math. Soc. 365, 10 (2013), 5035-5050.

[13] A. KRISTALY, Sharp uncertainty principles on Riemannian manifolds: the influence of curvature, to appear in Journal de Mathématiques Pures et Appliquées, (2017), arXiv:1311.6418v4.

[14] Yu. A. Kuperin, R. V. Romanov, H. E. Rudin, Scattering on the hyperbolic plane in the Aharonov-Bohm gauge field, Lett. Math. Phys. 31, 4 (1994), 271-278.

[15] A. Laptev And T. WeidL, Hardy inequalities for magnetic Dirichlet forms, Mathematical Results in Quantum Mechanics (Prague, 1998), Oper. Theory Adv. Appl., vol. 108, Birkhäuser, Basel, (1999), 299-305.

[16] P. Niu, Y. Chen, AND Y. Han, Some Hardy-type inequalities for the generalized Baouendi-Grushin operators, Glasg. Math. J. 46, 3 (2004), 515-527. 
[17] K. SAndeep, C. Tintarev, A subset of Caffarelli-Kohn-Nirenberg inequalities in the hyperbolic space $\mathbb{H}^{N}$, Ann. Mat. Pura Appl. 196, 6 (2017), 2005-2021.

[18] Q. YANG, D. SU, Y. Kong, Hardy inequalities on Riemannian manifolds with negative curvature, Commun. Contemp. Math. 16, (2014), 1350043, 24 pp. 